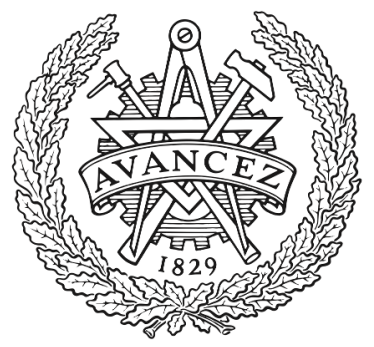

CHALMERS

UNIVERSITY OF TECHNOLOGY

\title{
Inclusive design strategies to enhance inclusivity for all in public transportation - A case study on a railway station
}

Downloaded from: https://research.chalmers.se, 2023-04-26 09:55 UTC

Citation for the original published paper (version of record):

Nybacka, M., Osvalder, A. (2019). Inclusive design strategies to enhance inclusivity for all in public transportation - A case

study on a railway station. Advances in Intelligent Systems and Computing, 824: 1689-1698.

http://dx.doi.org/10.1007/978-3-319-96071-5_173

N.B. When citing this work, cite the original published paper. 


\title{
Inclusive design strategies to enhance inclusivity for all in public transportation - A case study on a railway station
}

\author{
Moa Nybacka ${ }^{[1]}$ and Anna-Lisa Osvalder ${ }^{[1]}$ \\ ${ }^{1}$ Chalmers University of Technology, Gothenburg, Sweden \\ alos@chalmers.se
}

\begin{abstract}
The way traffic information is presented is not optimal for all travellers despite that, transportation systems should be accessible to everyone. The purpose of this study was to investigate how people with various disabilities perceive, experience and need audible and visible traffic information when travelling, especially at railway stations. The goal was to propose ideas on how to improve the accessibility of traffic information. Elderly and people with hearing or visual impairment were studied using online surveys. Observations were also made at the central railway station in Gothenburg, Sweden. The results showed various user demands on how traffic information should be presented to be available and suitable. Visual information screens, mobile phones and spoken information were the mostly used information sources. In general, there are too few information screens and the applications on mobile phones are updated too slowly. During traffic disturbances there are too many announcements and the quality is perceived as poor. The background noise is noticed as too loud. To conclude, it is outmost important to include user experience in the design decisions regarding information flow. The following strategies are proposed for improvement: The number and placement of visual screens should be increased, the content, length and formulations of the audial announcements should be revised, implementation of attention signals should be introduced to alert travellers of new information, and mobile applications should achieve better coherence with the visual and spoken information sources. Quiet zones should be investigated to offer reduced background noise.
\end{abstract}

Keywords: Traffic information announcements, audible information, disabled

\section{Introduction}

This study is part of a larger research project initiated by the Swedish Transport Administration in collaboration with Chalmers University of Technology in Gothenburg, Sweden. The focus of the project was on how spoken information at travel centres should be accessible for all people. The research project was carried out in Sweden in early 2018 with the aim to assess how different user groups perceive, experience and need spoken information at travel centres, especially at railway stations [1][2]. The purpose of this study was, through application of inclusive design theories, to propose ideas for strategies to improve the suitability of travel centres for 
all travellers, with special emphasis on elderly and people with visual or hearing impairments.

\section{Theoretical background}

\section{$2.1 \quad$ Elderly}

The user group, elderly, can be defined based on different parameters as chronological age (+65 years), appearance (grey hair or wrinkles), or based on their behaviour or functional status. Two common ways of categorization are to state if the person is working or not and if the person has any age-related life role like being a grandparent or retiree.

This user group is growing and has very varying abilities. Some people within this group are very independent while others need help and support to manage their daily tasks. The older an individual gets, the higher the risk of diseases that can affect different physical and cognitive abilities. Impaired mobility is common and makes the elderly move slower and more unstable. Due to eyesight deterioration it becomes harder to adapt the sight to various distances and light intensities. The colour vision also deteriorates and the sensitivity of glare increases. The cognitive degradation involves, among other things, that learning new things becomes more difficult, the memory gets weaker and the reaction time becomes longer [3][4]. The decision making is also prolonged. The selective and targeted attention of elderly people may also deteriorate, which gives concentration difficulties if disturbing stimuli occur. This may cause problems to sort out relevant information [5].

\subsection{Visual impairment}

There are several reasons for why people suffer from impaired vision. The most common reasons are different kinds of eye diseases and age-related degeneration. All visually impaired persons are seeing in their own individual and limited way. The symptoms vary, from a slightly blurred vision to partial vision loss to complete blindness. Reading becomes harder, therefore receiving spoken information is more vital. Some visually impaired uses Braille for communication while others just need to read up close or use a magnifying glass. Many people within this user group also have problems orientating in new, unfamiliar environments. The use of different aids such as technical devices giving spoken information or the white cane is needed [6].

\subsection{Hearing impairment}

The most common reasons for hearing impairment are hearing degradation due to age and exposure to noisy environments. It may also be innate. Everybody who suffers from a hearing impairment, regardless of its degree, experience to some extent that the sound becomes weaker and more indistinct, some frequencies disappear and the noise is distorted. It becomes more difficult to perceive what others say and to separate different sounds from each other [7]. This also means that the cognitive ability to sort out important sounds is diminished and different sounds are mixed 
together to an unrecognizable sound mass, i.e. a high background noise disturbs the speech intelligibility. The relationship between background noise and speech can be described as the so-called signal-to-noise ratio, which should be at least $25 \mathrm{~dB}$ for an elderly person with hearing impairment, but only $10 \mathrm{~dB}$ for a normal hearing person [7]. People with hearing impairment are vastly dependent on getting visual information as the main information source.

\subsection{Problems for elderly and disabled people within public transportation}

Depending on what disability the passengers have, they are affected to different extent and in various ways by traffic disturbances. People with impairments choose other travelling options than public transportations since they do not trust that the whole trip will function as it should. It is important to continue to improve the shortcomings of accessibility to restore the trust of people with disabilities. The decisive factor for elderly and people with disabilities to feel safe and capable of completing a journey is to be able to identify which vehicle they should choose and where to get on and off the vehicle [8]. Ronström [9] mention that older travellers do not want to show their possible uncertainty about independently traveling with public transportation. They highly value safety both on board the vehicles and at the stops/stations.

People with visual impairment may have difficulties to orientate in new, unknown environments. They also may have difficulties with perceiving visual information, which becomes evident when travelling by public transportation. The prerequisites for people with visual impairment to travel independently entail that spoken information is of great importance for them to receive significant information. However, large individual differences exist and depending on the situation and the person's current state, the possibilities for traveling independently can still vary greatly [6]. This means that it is not only circumstances regarding practical accessibility that need to be taken into consideration, but also the individual prerequisites as well as the environment of the public transportation which often can be perceived as stressful.

People with hearing impairment travelling with public transport have difficulties perceiving spoken announcements. The high background noise from traffic also disturbs the speech intelligibility. This user group demand visual information via information boards and TV screens. The information should be consistent with the spoken information given through public announcement (PA) systems to provide the passenger security and reassurance. The Traffic Administration [10] in Sweden states that needs of children, elderly and people with disabilities should be prioritized and information should be available and accessible to these user groups in society.

\section{Methods}

Three online surveys were conducted, one survey to gather the opinions from people with impaired vision, one to collect the opinions from people who suffer from hearing impairment and one to collect elderly people's opinions. These people might have specific needs and prerequisites to perceive and comprehend the information. The purpose of the online surveys was to get a broad picture about how these people, at 
various ages and geographical spread experience spoken information at railway stations. The surveys focused on how the spoken information was perceived by the respondents, how it affected them, if the information was useful and fulfilled their needs as well as how they wanted to receive information about their trip. All three surveys consisted of 19 individual questions. A couple of questions dealt with grading the perception and experience of the spoken information from very good to very bad, or from very high to very low. It took approximately 10 minutes to answer the surveys and it was anonymous.

The surveys were distributed as a link by email to contact persons representing different districts of several retirement unions, the Swedish Association of Visually Impaired and the Swedish Association of Hard of Hearing People, the nationwide covenant for visual impaired people and audible impaired people respectively. The surveys were available online for approximately three weeks and one reminder was sent to all groups by email after one week.

Observations were made during five hours at the central railway station in Gothenburg with the purpose to study passengers and visitors behaviour during different traffic announcements through the stations PA-system. Another purpose was to study the spoken information sources that the railway station provided, the socalled speaking machines as well as their placement and use. The speaking machines are boxes that give spoken information on the users' demand and exist as both small and large devices (Figure 1). Short questions were also asked to different travellers regarding the use and perception of the different sources of information.

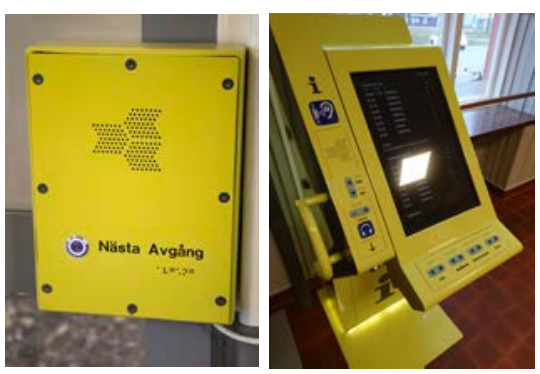

Fig. 1. Speaking machines that give spoken information on users' demand. The small device to the left and the large device to the right.

\section{$4 \quad$ Results}

\subsection{Audibly impaired}

The survey sent to the Swedish Association of Hard of Hearing People got 104 respondents with an even geographical spread, with emphasis on the major city regions. All age groups were represented, but most respondents were in the age group 40-55 years. Most respondents (34\%) thought the volume of the announcements was rather good. However, the respondents considered the volume as more high than low. The number of announcements during normal traffic conditions was considered as 
sufficient while the number of announcements during traffic disturbances was considered by the majority as too many.

Most respondents (84\%) thought that there are much disturbing background noises at the railway stations. The main sources of noise are trains, other traffic and other passengers. Other statements concerned the announcements' sound quality, the speed of the speaker's voice, the acoustics of the railway station, wind, closing signal of train doors as well as baggage wheels against asphalt. Important information that most respondents with hearing impairment required through spoken announcements was traffic disturbances and changes, like changes of platforms and departure time. Information about departures was desired by $14 \%$ while $35 \%$ did not want any spoken information at all.

The information sources that this user group uses can be seen in figure 2. They mostly use visual information screens and mobile phones. The majority almost never used the speaking machines or the advertiser machines. Advertiser machines give automatically spoken information about the next departing train at the platform and are solely used for commuter trains in the region of Stockholm. Around $60 \%$ feels that information sources are missing in different areas of the railway station and $65 \%$ points out that information is missing at the platforms. Cafes and restaurants are other areas at the railway station where passengers lack various information sources. More and larger displays are also desired by most respondents and $8 \%$ asked for clearer and more relevant information. Few respondents (3\%) also asked for hearing loops.

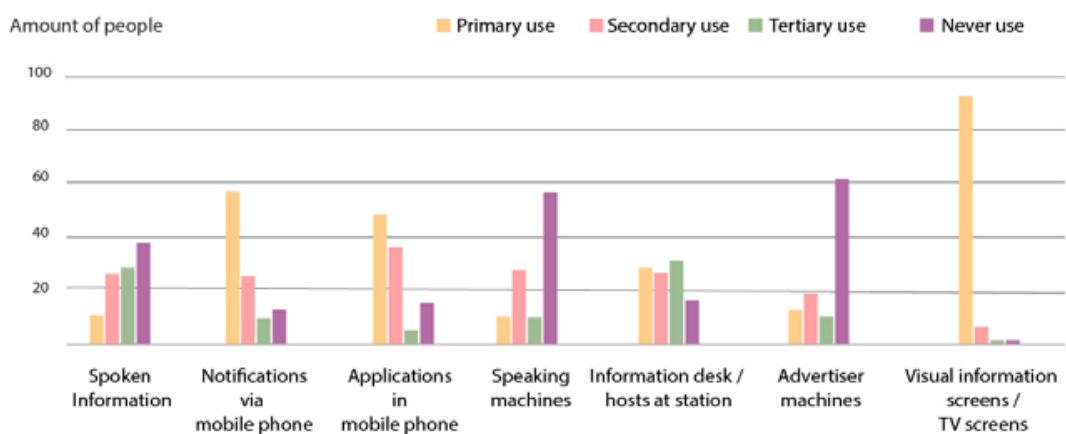

Fig. 2. The information sources primarily, secondarily, tertiarily and never used by the respondents of the survey sent to the Swedish Association of Hard of Hearing People.

To summarize, the perceived disadvantages concerning the spoken information was that it was hard to hear and perceive due to its low sound quality and the many disturbing background sounds at the railway station. Most respondents did not perceive any advantages with the spoken information. On the other hand, $24 \%$ mentioned that the announcements, especially the signal before the message, made them pay attention that something has changed and 10\% thought that the spoken information probably is good for people that can hear it. 


\subsection{Visibly impaired}

The survey that was sent to the Swedish Association for the Visually Impaired got 32 respondents, mostly from the major city regions of Gothenburg, Stockholm and Malmö. The spread between the age groups were even. Most respondents perceived the volume as neither too loud nor too low. The number of announcements was also perceived as good, neither too many nor too few, during normal traffic. During disturbances the number of announcements was by the majority perceived as too few. Most of the respondents thought there were many disturbing background sounds. The most disturbing sounds were those of trains and other passengers. Other disturbing sounds were the echo from the railway stations' acoustics, the sound quality of the speakers, the speed of the speaker voice and the signals from closing train doors.

Important information that should be given as spoken information are traffic changes and disturbances as well as departure information. The sources that primarily are used are spoken information through the railway stations' speakers and mobile phones. The sources that are used the least are the speaking machines, advertiser machines and visual information screens (Figure 3). When asked if the respondents lack information sources, 55\% stated that they missed information sources at various areas of the railway station, mostly at the platforms but also at main entrances, in waiting halls, tunnels as well as on pedestrian bridges. Clearer and larger information screens were also desired and the placement of the screens should be in eye height to be easier to read at a closer distance. Information screens were also desired at cafes and restaurants. Information desks for personal service were also desired.

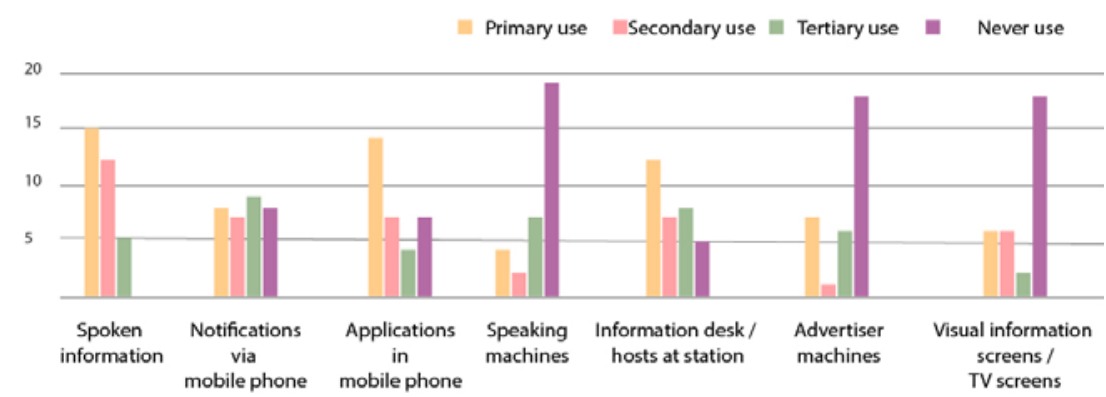

Fig. 3. The information sources primarily, secondarily, tertiarily and never used by the respondents of the survey sent to the Swedish Association of the Visually Impaired.

Around $42 \%$ of the respondents thought that no disadvantages existed concerning the spoken information but highlighted that the sound quality should be improved. About $16 \%$ desired personal service since the number of announcements was perceived as disturbing and the information sometimes too long and irrelevant. The advantages with the spoken information were according to $52 \%$ that they are automatic and that you do not need to search for the information. Approximately 39\% said that the spoken information is directly crucial for them to be able to travel independently and $26 \%$ mentioned that the spoken information gives them a secure feeling. 


\subsection{Elderly}

The survey sent to different Swedish retirement unions got 89 respondents in total. The age spread between 66 to above 86 years, but most respondents were between 66 and 75 years. The geographical spread of the respondents was mostly from the regions of the larger cities Gothenburg and Stockholm. Around $76 \%$ of the respondents use information screens while the rest either uses the mobile phone or spoken information. Of the respondents, $63 \%$ think the spoken information should be improved and that there are many factors that make it hard to perceive even if the volume is satisfying. Some of the other factors that were mentioned were the station's acoustics and that the announcements are spoken too quickly and with a monotonous voice. Still, $37 \%$ are satisfied with the spoken information as it is designed today. Most respondents (36\%) said that the announcements were neither difficult nor easy to perceive, whereas (33\%) thought the announcements were more difficult than easy to perceive. Elderly people desire personal service to feel secure, since the announcements are perceived as unclear and the background noise disturbing. Elderly perceive the volume of spoken information as low and the quality as poor. The information sources elderly primarily use is a combination of visual information screens and spoken information. Often this user group feels unconfident about their ability to perceive information correctly and sometimes want personal service to confirm the information they have perceived through visual screens and spoken information.

\subsection{Site observations}

At the central railway station in Gothenburg there were many visual information screens. There were also two smaller speaking machines and one larger speaking machine, all of which were difficult to find. The placement of the large speaking machine was not at a main entrance, which would be optimal in relation to its purpose. Most passengers went to the largest central information screen at their arrival to the railway station to confirm the departure time, platform number and get information concerning delays or changes.

During the site observation, short questions were asked a few passengers regarding the different information sources available at the station. Most passengers preferred the visual information screens as primary information source. Spoken information, on the other hand, was preferred during traffic disturbances since it was believed to be the fastest and easiest way to receive information. However, the number of announcements was also considered to be too many and non-informative during traffic disturbances. Some of the announcements that were made were over explanatory, for example "SJ High speed train, service number 525, with departure time 12:47, to Copenhagen airport, Copenhagen, has been cancelled and replaced by bus. Bus departs from Nils Ericson Terminal, at the north end of the central station, close to platform 16. Which gate your bus departs from can be seen at the terminal monitors. " Information about from where the replacement bus departs is given in three different ways: the name of the terminal (Nils Ericson Terminal), a direction (north end) and a landmark (platform 16). 


\section{Discussion}

The results show that most people plan their trip in advance by buying tickets online. They arrive at the station close to the train's departure time. At the railway station they most frequently use visual information screens, mainly the biggest screen they can find, to confirm travel information about departure time, platform number and changes. Mobile applications are also frequently used while spoken information is mainly appreciated for alertness and essential information during traffic disturbances.

People with disabilities often need more time to find necessary information and locate right platform, train or other transportation options. If late changes are made, they have difficulties to perceive the information and locate new platforms or replacement busses. Most elderly want personal service to feel secure, not having to study information screens or listen to announcements since they are perceived as complex and hard to extract relevant information from. Elderly want relevant information through the announcements and find that relevant information is easier to receive through personal service by station hosts or at information desks. Younger and middle-aged passengers prefer to receive traffic information via their mobile phones since they then only get the information that is relevant for them and do not have to listen to all announcements.

Elderly and people who have mobility problems need more time to find necessary information and locate right train and platform. To facilitate for these people to be efficiently informed, more screens for visual information are required and should be located at various places. First and foremost, at the platforms.

Visually impaired people primarily use spoken information given via the railway station's PA-system as well as via mobile phones. They desire more spoken information that also should be clearer. They have problems perceiving the spoken information due to disturbing background sound. The sound quality is also perceived as poor. They almost never use speaking machines since these are difficult to find and are perceived difficult to use. Websites and mobile applications with traffic information are also thought to be updated too slowly. They require better placing of information signs and a layout that enhance the readability as well as intelligibility. They highlight the lack of spoken information at the platforms and the importance of a uniform design of the ground structure for enhanced localization when using white canes. They would appreciate if train companies could offer a mobile application with a description of where to stand on the platform to reach their seat when boarding.

Hearing impaired people say that the most disturbing background sounds are trains passing by, other traffic and fans. This thought is also shared by the visually impaired. Other passengers are also producing disturbing background sound. The signal before an announcement is often perceived but not the message itself. This signal gives the passenger an indication that new information is available or that changes have been made. They then often check other information sources to get the needed information to pursue with their trip. Some respondents propose silent zones to be able to hear and perceive the spoken information given through the PA system since the background noise would be reduced. They perceive the large number of announcements during traffic disturbances as annoying; they would rather get only a sound or light signal 
that new information is available. Detailed information could be received through the dynamic information screens at several placements as well as on mobile phones.

\section{$6 \quad$ Concluding strategies}

To conclude, the results show that a variety of inclusive design strategies are needed to enhance the perception, experience and inclusivity of spoken information for all people at railway stations. It is vital to remember to involve users with various disabilities in all parts of both design and redesign processes.

One strategy is to take into consideration that disabilities can be situation based, which means that appropriate design guidelines should be used for improved comprehension, mobility and user experience related to the specific environment. One way to approach this is to reflect on the amount and clarity of visual and spoken information. Correct traffic information should be provided at the right time and the traffic information should be adapted to as many travellers as possible, regardless of age and disability. This indicates that the layout and number of announcements should be investigated and the content, length and formulations of the announcements should be revised. A common mistake when redesigning an interface is to reduce information to decrease user overloaded. Here, it is not the amount of information that is decisive but the relevance and exposition of it. A redesign of the announcements should not affect the information and aggravate a completion of the passengers' journey.

A second strategy is to reflect on the amount of information sources and their clarity. The information sources that exist today should be evaluated and additional information sources for different user groups need to be developed to meet the users' contrasting demands. Hearing impaired people mostly use visual information screens whereas visual impaired often need personal service. Mobile phones are used to a large extent to search for traffic information. The study shows, for example that the visual information screens should be increased in number and their readability and intelligibility should be enhanced.

A third strategy is to investigate how more reliable traffic forecasts during disturbances can be distributed, so they only include relevant information. The passengers' attitude towards the train companies and the Swedish Transport Administration will be improved if only significant information will be provided. A consequence of this is that fewer announcements are made, which in turn reduces the experience of the announcements as ambiguous and their credibility increases.

A fourth strategy is to explore the existing mobile applications and websites, which provide traffic information. This in terms of their strengths, weaknesses and development opportunities to achieve greater coherence with the visual and spoken information sources at the railway station. It should also be investigated whether they may replace or supplement other information sources. Potential further development of mobile applications and websites may be using human factors' design guidelines. An attention signal, which could be sound, light or both, could make people aware of that new or updated traffic information is available. 
A fifth strategy is to investigate existing attention signals concerning what type of signals most people respond to and benefit from. Emphasis should be put on how the different user groups with special needs respond to the various signals. How should an attention signal function and be designed are also issues for further investigations.

A sixth strategy is to explore the possibility of introducing quiet zones. This would be zones with reduced background noise, which could strengthen the spoken information. A possible implementation of quiet zones may also affect how the railway station is perceived and experienced as a less stressful environment. This especially regards elderly and people with hearing or visual impairment. The quiet zones could improve the understanding and perception of the announcements and thereby make these people feel more safe and confident.

\section{References}

1. Nybacka, M., Osvalder, A-L. Spoken traffic information at railway stations - A behavioural study on how spoken information is perceived and should be designed for improved customer experience. Chalmers University of Technology. Gothenburg, Sweden. In Swedish. (2018).

2. Osvalder A-L., Nybacka, M.: Different user groups' perception, experience and need of spoken information in public transportation spaces. AHFE. Chalmers University of Technology. Gothenburg, Sweden. (2018)

3. Johansson, B.: Aging's effect on memory and thought processes. In When memory fails About dementia and dementia resembling conditions. (pp. 43-55). Andersson, D et al. (eds.). Spri och Svenska Läkaresällskapet. Stockholm. In Swedish. (1994).

4. Groeger, John A. (2000). Understanding driving: Applying cognitive psychology to a complex everyday task. Hove. Psychology Press. (2000).

5. Levin, L. (eds.), Dukic, T., Heikkinen, S., Henriksson, P., Linder, A., Mårdh, S., Nielsen, B., Nygårdhs, S., Peters, B.: Elderly in the transport system - Mobility, design and training problems. VTI report 593. Proj. nr:12478/12312. Ref nr: 2007/0533-23. In Swedish. (2007).

6. Swedish Association of the Visually Impaired Homepage, In Swedish. http://www.srf.nu, last accessed 2017/12/15.

7. Swedish Association of Hard of Hearing People Homepage, In Swedish. https://horsellinjen.se, last accessed 2017/10/30.

8. Metz, D.H.: Mobility of older people and their quality of life, Transport Policy, vol. 7, Issue 2, pp. 149-152. (2000).

9. Ronström, O. (eds.): Alert seniors and popular culture. Carlssons Bokförlag, Stockholm, Sweden. In Swedish. (1998).

10. Traffic Administration Guidelines Accessibility for children, elderly and disabled people. Traffic Administration Stockholm County Council, Strategic development, Sustainable development. In Swedish. http://www.sll.se, last accessed 2017/12/01. 